\title{
Hábitos alimentarios y factores de riesgo para patologías crónico-degenerativas en universitarios
}

\section{Eating habits and risk factors for chronic-degenerative pathologies in university students}

\author{
DOI: $10.46932 / s f j d v 3 n 1-119$
}

Received in: January $20^{\text {th }}, 2022$

Accepted in: February $1^{\text {st }}, 2022$

\section{Rosa Martha Velasco Martínez}

Dra. En Ciencias de la Salud. PTC Nutriología

Institution: Universidad Autónoma de Chiapas, Facultad de Medicina Humana Dr. Manuel Velasco

Suárez C-II, Calle central entre 10 y 11 sur. SN CP: 29090

Correio eletrônico: oro_vel@ hotmail.com

\section{Zally Patricia Mandujano Trujillo}

Mtra. En Docencia en Ciencias de la Salud. PTC anatomía y fisiología

Institution: Universidad Autónoma de Chiapas, Facultad de Medicina Humana Dr. Manuel Velasco

Suárez C-II, Calle central entre 10 y 11 sur. SN CP: 29090

Correio eletrônico: zallym@hotmail.es

\section{Sonia Rosa Roblero Ochoa}

Dra. en Ciencias para la Salud. PTC inmunología

Institution: Universidad Autónoma de Chiapas, Facultad de Medicina Humana Dr. Manuel Velasco

Suárez C-II, Calle central entre 10 y 11 sur. SN CP: 29090

Correio eletrônico: sroblero1504@hotmail.com

\section{Tomasa de los Ángeles Jiménez Pirrón}

Presidente del comité de bioética, PTC de bioquímica. Dra. en Tecnologías de la Educación. Institution: Universidad Autónoma de Chiapas, Facultad de Medicina Humana Dr. Manuel Velasco

Suárez C-II, Calle central entre 10 y 11 sur. SN CP: 29090

Correio eletrônico: angeles_pirron@ hotmail.com

\section{Itzel Castro Padilla}

Médico

Profesor de asignatura, bioquímica

Institution: Universidad Autónoma de Chiapas, Facultad de Medicina Humana Dr. Manuel Velasco

Suárez C-II, Calle central entre 10 y 11 sur. SN CP: 29090

Correio eletrônico: Itzel.castro@unach.mx

\section{RESUMEN}

Introducción: Las enfermedades crónico-degenerativas representan una causa de morbimortalidad ascendente. Identificar los principales factores de riesgo en poblaciones específicas permite realizar intervenciones específicas y el diagnóstico oportuno de ellas. Objetivo: Identificar los principales factores de riesgo y hábitos alimentarios para el desarrollo de Enfermedades No Transmisibles (ENT) en universitarios. Método: Estudio descriptivo, transversal. La muestra correspondió a alumnos de (1 y 8 semestre) de la licenciatura de medicina. El instrumento incluyó datos de la historia clínica, como cifras tensionales por JN8 (Eight Joint National Committe), IMC, ICC y los parámetros de composición corporal 
de InBody (J1001). Resultados: De los participantes, 58\% ( $\mathrm{n}=51)$ correspondieron al género femenino. Los patrones de composición corporal mostraron un claro dimorfismo sexual. El $35.3 \%$ ( $\mathrm{n}=31$ ) presentó sobrepeso u obesidad. La principal práctica alimentaria perjudicial fue el consumo de comida rápida (97.7\% n=86). El 15.9\% ( $\mathrm{n}=14)$ presentó cifras tensionales altas, el 18.9\% ( $\mathrm{n}=7)$ del género masculino presentó prehipertensión y 58\% $(\mathrm{n}=51)$ reportó más de dos antecedentes heredofamiliares crónicos degenerativos. Conclusiones: El número de estudiantes con datos de enfermedad hipertensiva son alarmantes, la muestra siguió la tendencia nacional al sobrepeso en hombres y obesidad en mujeres. Los parámetros de ICC observados nos permiten inferir una mayor frecuencia en alta adiposidad abdominal. El consumo de alimentos con elevado contenido de sodio, grasas trans y bajo omega-3 aunado al menor consumo de frutas y verduras también fue mayor a lo esperado. Se encontró una correlación moderada entre IMC y PGC, PAS y MME.

Palabras clave: composición corporal, sobrenutrición, factores de riesgo cardio metabólicos.

\begin{abstract}
Introduction: Chronic-degenerative diseases represent a cause of increasing morbidity and mortality. Identifying the main risk factors in specific populations allows for specific interventions and their timely diagnosis. Objective: To identify the main risk factors and eating habits for the development of NonCommunicable Diseases (chronic degenerative) in university students. Method: Descriptive, crosssectional study. The sample corresponded to students of the medical school. The instrument included data from the clinical history, such as JN8 (Eight Joint National Committee) for blood pressure, BMI, WHR, and InBody (J1001) body composition parameters. Results: Of the participants, $58 \%(\mathrm{n}=51)$ were female. Body composition patterns showed clear sexual dimorphism. 35.3\% $(n=31)$ were overweight or obese. The main harmful eating practice was the consumption of fast food $(97.7 \% \mathrm{n}=86) .15 .9 \%(\mathrm{n}=14)$ presented high blood pressure figures and 58\% $(n=51)$ reported more than two chronic degenerative family histories. A moderate correlation was found between BMI and PGC, SBP and MME. Conclusions: The number of students with hypertensive disease data is alarming, the sample followed the national trend of overweight in men and obesity in women. The observed WHR parameters allow us to infer a higher frequency in high abdominal adiposity. Consumption of foods high in sodium, trans fats, and low in omega-3s, coupled with lower consumption of fruits and vegetables, was also higher than expected.
\end{abstract}

Keywords: body composition, overnutrition, cardiometabolic risk factors.

\title{
1 INTRODUCCIÓN
}

Las enfermedades no transmisibles (ENT) o enfermedades crónico degenerativas representan al menos el $75 \%$ de las defunciones anuales a nivel mundial, sobre todo en países con medianos y bajos ingresos. Estas enfermedades han sido claramente asociadas a factores genéticos y también a hábitos que aumentan el riesgo cardiovascular, tales como sobre ingesta calórica con el potencial de producir patologías metabólicas (sobrepeso y obesidad, diabetes mellitus, hipertensión arterial), toxicomanías (tabaquismo y alcoholismo) y sedentarismo. Todas estas patologías se ven interrelacionadas, afectando de gran manera a aquellos grupos vulnerables donde llevar un estilo de vida saludable no es factible ante la falta de recursos económicos, es aquí donde se deben enfocar las acciones de prevención, ya que la edad de debut de pacientes crónico-degenerativos suele ser más prematura en la actualidad. 
Las ENT representan una carga importante sobre salud pública y a medida que la transición epidemiológica ocurre, los gastos sanitarios aumentan año con año, por ello, esta situación se incluyó en los Objetivos de Desarrollo Sostenible para el 2030, persiguiendo conseguir que la mortalidad asociada a las ENT disminuya en un 33\% (Organización Mundial de la Salud, 2021). En México, las tres principales causas de muerte corresponden a enfermedades cardiovasculares (218 885, 20.2\%), COVID-19 (201 163, $18.5 \%)$ y diabetes mellitus (151 214, 13.9\%). (Instituto Nacional de Estadística y Geografía, 2021). En el caso de Chiapas, Jiménez y Núñez (2016) identificaron como las principales causas de muerte a las enfermedades del corazón, la diabetes mellitus y enfermedades cerebrovasculares previo al inicio de la pandemia.

Los adultos jóvenes son un grupo de riesgo importante, muchos de ellos han iniciado su vida universitaria. Este cambio se ve vinculado al desarrollo de malas prácticas alimenticias como ayuno prolongado, ingesta de comida rápida, el sedentarismo y la adquisición de toxicomanías, los cuales son factores de riesgo modificables. El tipo de alimentos consumidos juega uno de los papeles más importantes, observando que por lo general el consumo de grasa animal predomina sobre la vegetal (Cruz et al, 2019). Entre los factores de riesgo no modificables documentados para el desarrollo de ENT, se ha observado que el sobrepeso y la obesidad en el estado de Chiapas, prevalece en el $70.9 \%$ para hombres y 60.6\% para mujeres en el grupo de mayores de 20 años (Centro Nacional de Programas Preventivos y Control de Enfermedades). La obesidad e hipertensión son los antecedentes heredofamiliares más importante (hasta en un 40\%) en estudios similares (Gómez et al, 2018).

De acuerdo con la ENSANUT (Basto et al, 2015), estima que al menos 3.3\% de los mexicanos diagnosticados con diabetes, pertenecen al grupo comprendido entre 20-29 años, así como 5.4\% de los diagnosticados con hipertensión arterial (Campos et al, 2018). En cuanto a sobrepeso y obesidad, se encontró que su prevalencia en adolescentes es de 33.6\% y en los adultos 72.5\% (Shamah et al, 2019). Estos valores representan un problema con tendencia ascendente, que de identificarse oportunamente puede evitar no solo menor carga a la salud pública, si no mayor calidad y esperanza de vida, siendo imperante el estudio de las poblaciones vulnerables para la optimización de estrategias preventivas.

\section{MATERIAL Y MÉTODO}

Estudio de diseño mixto, de corte transversal, muestreo a conveniencia. El universo correspondió a los alumnos matriculados en el primer y último semestre de la licenciatura de medicina, incluyendo a todo aquel que aceptó participar en el estudio. La muestra fue de 88 estudiantes. El instrumento fue de elaboración propia, aplicado mediante una entrevista y toma de datos antropométricos con dispositivo InBody (J1001). Las cifras de tensión arterial fueron interpretados acorde a los parámetros de JNC 8, los 
valores de referencia de IMC a los de la OMS y los de composición corporal conforme al dispositivo utilizado. El análisis estadístico descriptivo y correlacional se realizó en el programa estadístico SPSS versión 25.

\section{RESULTADOS}

De los 88 sujetos de estudio, $58 \%(\mathrm{n}=51)$ correspondieron al género femenino, el restante, $42 \%$ $(\mathrm{n}=37)$ al masculino. El 61.4\% $(\mathrm{n}=54)$ de los estudiantes pertenecieron al grupo de edad comprendido entre 21 a 25 años, $37.5 \%(n=3)$ entre 18 y 20 años.

Tabla 1. Estadísticos descriptivos de composición corporal y somatometría

\begin{tabular}{|c|c|c|c|c|c|}
\hline & $\mathrm{N}$ & Mínimo & Máximo & Media & $\mathrm{DE}$ \\
\hline PAS en $\mathrm{mmHg}$ & 88 & 90 & 146 & 110.25 & 12.994 \\
\hline Cintura en centímetros & 88 & 61 & 105 & 79.051 & 9.6243 \\
\hline PAD en mmHg & 88 & 50 & 100 & 68.76 & 8.992 \\
\hline ACT en litros & 88 & 21.6 & 48.5 & 33.547 & 6.9377 \\
\hline PGC en kilogramos & 88 & 17.8 & 52.2 & 29.525 & 9.9175 \\
\hline MME en kilogramos & 88 & 16 & 39.1 & 26.188 & 5.9712 \\
\hline MGC en kilogramos & 88 & 12.6 & 51.2 & 19.518 & 8.9209 \\
\hline Proteínas en kilogramos & 88 & 6 & 13.6 & 9.326 & 2.0109 \\
\hline Minerales en kilogramos & 88 & 1.46 & 10.55 & 2.6561 & 1.15539 \\
\hline ICC & 88 & 0.68 & 1.22 & 0.99019 & 0.102152 \\
\hline
\end{tabular}

PAS: presión arterial sistólica. PAD: presión arterial diastólica. ACT: agua corporal total. PGC: porcentaje de grasa corporal. MME: masa de músculo esquelético. MGC: masa grasa corporal. ICC: índice cintura-cadera.

La tabla 1 presenta los parámetros de composición corporal y antropometría en un análisis general, se observó un mayor rango en el parámetro correspondiente a presión arterial sistólica derecha con un rango de $56 \mathrm{mmHg}$ (DE 12.9), seguido por perímetro de cintura con un rango de $44 \mathrm{~cm}$ (DE 9.6). El parámetro con la menor DE correspondió a índice cintura cadera (0.10).

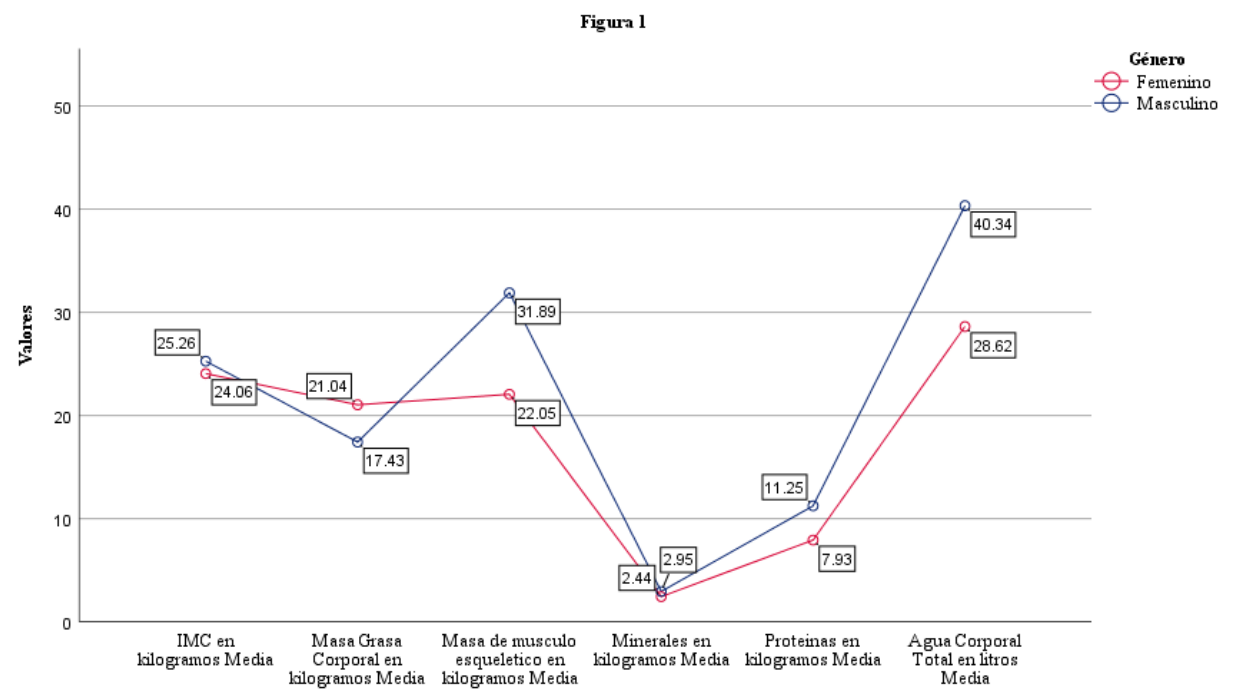


La figura 1 representa a las medias en relación a parámetros de composición por género, únicamente el parámetro de masa grasa corporal media reportó valores mayores en el femenino. Los parámetros de masa de músculo esquelético y agua corporal total en el masculino presentaron valores mas alejados y superiores de la media para el femenino.

Tabla 2. IMC por género

\begin{tabular}{llll}
\hline & Femenino & Masculino & Total \\
\hline Bajo & $2 \%(\mathrm{n}=1)$ & & $1.1 \%(\mathrm{n}=1)$ \\
Normal & $62.7 \%(\mathrm{n}=32)$ & $64.9(\mathrm{n}=24)$ & $63.5 \%(\mathrm{n}=56)$ \\
Sobrepeso & $23.5 \%(\mathrm{n}=12$ & $16.2(\mathrm{n}=6)$ & $20.5 \%(\mathrm{n}=18)$ \\
Obesidad I & $7.8 \%(\mathrm{n}=4)$ & $18.9 \%(\mathrm{n}=7)$ & $12.5 \%(\mathrm{n}=11)$ \\
Obesidad II & $3.9 \%(\mathrm{n}=2)$ & & $2.3 \%(\mathrm{n}=2)$ \\
Total & $100 \%(\mathrm{n}=51)$ & $100 \%(\mathrm{n}=37)$ & $100 \%(\mathrm{n}=88)$ \\
\hline
\end{tabular}

En relación con el diagnóstico de sobrepeso y obesidad, el género femenino presentó mayor variabilidad. El $63.60 \%(\mathrm{n}=56)$ de los sujetos estudiados obtuvieron parámetros dentro de la normalidad.

Tabla 3. Frecuencia de hábitos alimenticios negativos

\begin{tabular}{lrrrrr}
\hline & Comida rápida & \multicolumn{1}{l}{$\begin{array}{l}\text { Alimentos } \\
\text { enlatados }\end{array}$} & \multicolumn{1}{l}{$\begin{array}{l}\text { Alimentos para } \\
\text { Microondas }\end{array}$} & $\begin{array}{l}\text { Comidas fuera } \\
\text { de casa }\end{array}$ & \multicolumn{2}{l}{$\begin{array}{l}\text { Refrescos } \\
\text { embotellados }\end{array}$} \\
\hline No consumen & $2.3 \%(\mathrm{n}=2)$ & $10.2 \%(\mathrm{n}=9)$ & $48.9 \%(\mathrm{n}=43)$ & $6.8(\mathrm{n}=6)$ & $14.8(\mathrm{n}=13)$ \\
1 a 3 por sem & $78.4 \%(\mathrm{n}=69)$ & $81.8 \%(\mathrm{n}=72)$ & $50 \%(\mathrm{n}=44)$ & $47.7 \%(\mathrm{n}=42)$ & $67 \%(\mathrm{n}=60)$ \\
4 a 7 por sem & $19.3 \%(\mathrm{n}=17)$ & $7.9 \%(\mathrm{n}=7)$ & $1.1 \%(\mathrm{n}=1)$ & $45.4 \%(\mathrm{n}=40)$ & $5.6 \%(\mathrm{n}=15)$ \\
Sem: Semana & & & & &
\end{tabular}

Conforme a lo observado, el hábito alimentario negativo más frecuente correspondió a un consumo excesivo de ingesta de comida rápida, el $97.7 \%(n=86)$ de los encuestados refieren incluirla en su alimentación de forma inadecuada, seguido por comidas fuera de casa con $93.2 \%(\mathrm{n}=82)$, ingesta de alimentos enlatados $89.8 \%(n=79)$, consumo de refrescos embotellados con $72.7 \%(n=75)$ y $51.1 \%(n=45)$ de alimentos para microondas.

Tabla 4. Frecuencias de grupos de alimentos consumidos

\begin{tabular}{|c|c|c|c|c|c|c|c|c|c|c|}
\hline & $\begin{array}{c}\text { Carnes y } \\
\text { huevo }\end{array}$ & Lácteos & $\begin{array}{c}\text { Embutid } \\
\text { os }\end{array}$ & Frutas & Verduras & Cereales & $\begin{array}{c}\text { Tubércul } \\
\text { os }\end{array}$ & $\begin{array}{c}\text { Endulzan } \\
\text { tes }\end{array}$ & $\begin{array}{l}\text { Mantequi } \\
\text { lla, } \\
\text { margarin } \\
\text { a }\end{array}$ & $\begin{array}{l}\text { Frutos } \\
\text { secos }\end{array}$ \\
\hline $\begin{array}{l}\text { No } \\
\text { consume } \\
\text { n }\end{array}$ & & $\begin{array}{l}3.4 \% \\
(\mathrm{n}=3)\end{array}$ & & $\begin{array}{l}2.3 \% \\
(n=2)\end{array}$ & $\begin{array}{l}3.4 \% \\
(n=3)\end{array}$ & $\begin{array}{c}17 \% \\
(n=15)\end{array}$ & $\begin{array}{l}13.6 \% \\
(n=12)\end{array}$ & $\begin{array}{l}27.3 \% \\
(n=24)\end{array}$ & $\begin{array}{l}40.9 \% \\
(n=36)\end{array}$ & $\begin{array}{l}23.9 \% \\
(\mathrm{n}=21)\end{array}$ \\
\hline $\begin{array}{l}1 \text { a } 3 \text { por } \\
\text { sem }\end{array}$ & $\begin{array}{l}35.2 \% \\
(\mathrm{n}=31)\end{array}$ & $\begin{array}{l}53.4 \% \\
(n=47)\end{array}$ & $\begin{array}{l}69.3 \% \\
(n=61)\end{array}$ & $\begin{array}{c}50 \% \\
(n=44)\end{array}$ & $\begin{array}{l}36.4 \% \\
(n=32)\end{array}$ & $\begin{array}{l}53.4 \% \\
(\mathrm{n}=47)\end{array}$ & $\begin{array}{l}73.9 \% \\
(n=65)\end{array}$ & $\begin{array}{c}67 \% \\
(n=59)\end{array}$ & $\begin{array}{l}59.1 \% \\
(\mathrm{n}=52)\end{array}$ & $\begin{array}{l}62.5 \% \\
(n=55)\end{array}$ \\
\hline $\begin{array}{l}4 \text { a } 7 \text { por } \\
\text { sem }\end{array}$ & $\begin{array}{l}64.8 \% \\
(n=57)\end{array}$ & $\begin{array}{l}43.2 \% \\
(n=38)\end{array}$ & $\begin{array}{l}30.7 \% \\
(n=27)\end{array}$ & $\begin{array}{l}47.7 \% \\
(n=42)\end{array}$ & $\begin{array}{l}60.2 \% \\
(n=53)\end{array}$ & $\begin{array}{l}29.5 \% \\
(n=26)\end{array}$ & $\begin{array}{l}12.5 \% \\
(n=11)\end{array}$ & $\begin{array}{l}5.7 \% \\
(n=5)\end{array}$ & & $\begin{array}{c}13.6 \% \\
n=12\end{array}$ \\
\hline
\end{tabular}


La tabla 4 presenta las frecuencias de grupos de alimentos consumidos, se encontró que la totalidad de los alumnos consumen carnes y huevos con mayor frecuencia durante la semana. El siguiente grupo más consumido corresponde a las frutas y verduras. Los menos consumidos fueron endulzantes, mantequilla o margarina y cereales.

Tabla 5. Clasificación JNC8 de tensión arterial por género

\begin{tabular}{|c|c|c|c|}
\hline & Femenino & Masculino & Total \\
\hline Normal & $94.12 \%(\mathrm{n}=48=$ & $70.27 \%(\mathrm{n}=26)$ & 84.1 \\
\hline Prehipertensión & $5.88 \%(\mathrm{n}=3)$ & $18.92 \%(\mathrm{n}=7)$ & 11.36 \\
\hline \multirow[t]{2}{*}{ Hipertensión G1 } & & $10.81 \%(n=4)$ & 4.55 \\
\hline & & & $100 \%$ \\
\hline
\end{tabular}

El 84.1\% (n=74) de los estudiantes presentó cifras de normo tensión, sin embargo, se observó que el género masculino presenta un mayor porcentaje respecto a su categoría de prehipertensión $(18.9 \%$ n=7).

Tabla 6. Antecedentes heredofamiliares por género

\begin{tabular}{lrrr}
\hline \multicolumn{1}{c}{ Femenino } & \multicolumn{1}{c}{ Total } \\
\hline DM 2 & $74.51 \%(\mathrm{n}=38)$ & $81.08 \%(\mathrm{n}=30)$ & $77.27 \%(\mathrm{n}=68)$ \\
HAS & $62.75 \%(\mathrm{n}=32)$ & $70.27 \%(\mathrm{n}=26)$ & $65.90 \%(\mathrm{n}=58)$ \\
Dislipidemias & $31.37 \%(\mathrm{n}=16)$ & $24.32 \%(\mathrm{n}=9)$ & $28.40 \%(\mathrm{n}=25)$ \\
Obesidad & $50.98 \%(\mathrm{n}=26)$ & $45.95 \%(\mathrm{n}=17)$ & $48.86 \%(\mathrm{n}=43)$ \\
IAM & $21.57 \%(\mathrm{n}=11)$ & $18.92 \%(\mathrm{n}=7)$ & $20.45 \%(\mathrm{n}=18)$ \\
EVC & $5.88 \%(\mathrm{n}=3)$ & $13.51 \%(\mathrm{n}=5)$ & $9.09 \%(\mathrm{n}=8)$ \\
\hline
\end{tabular}

DM 2. diabetes mellitus 2. HAS: Hipertensión arterial sistémica. IAM: infarto agudo al miocardio. EVC: evento cerebrovascular

El 58\% de los estudiados ( $\mathrm{n}=51)$ reportó más dos antecedentes heredofamiliares. La diabetes mellitus es el más frecuente $(77.27 \% \mathrm{n}=68)$, seguido por HAS $(65.9 \% \mathrm{n}=58)$ y obesidad $(48.86 \mathrm{n}=43)$. Estos se presentan en el mismo orden de frecuencia para cada género.

Tabla 7. Toxicomanías y actividad física por género

\begin{tabular}{lccr}
\hline & Femenino & Masculino & \multicolumn{1}{c}{ Total } \\
\hline Tabaquismo & $3.92 \%(\mathrm{n}=2)$ & $10.81 \%(\mathrm{n}=4)$ & $6.8 \%(\mathrm{n}=6)$ \\
Consumo de alcohol & $50.98 \%(\mathrm{n}=26)$ & $56.76 \%(\mathrm{n}=21)$ & $53.4 \%(\mathrm{n}=47)$ \\
Actividad física & $49.02 \%(\mathrm{n}=25)$ & $56.76 \%(\mathrm{n}=21)$ & $52.27 \%(\mathrm{n}=46)$ \\
\hline
\end{tabular}

En lo que respecta a toxicomanías, el consumo de alcohol y tabaco es mayor en el género masculino que en el femenino. En el total de la población estudiantil, la toxicomanía más frecuente fue el consumo de alcohol $(53.40 \% \mathrm{n}=47)$ y el tabaquismo en el $6.8 \%(\mathrm{n}=6)$. El 52\% realiza algún tipo de actividad física $(\mathrm{n}=46)$. 
Tabla 8. Correlaciones en presión arterial y medidas antropométricas

\begin{tabular}{|c|c|c|c|c|c|c|}
\hline & IMC & PAS & PAD & $\mathrm{ICC}$ & PGC & MME \\
\hline IMC & 1 & $.440 * *$ & $.533 * *$ & $.426 * *$ & $.540 * *$ & $.374 * *$ \\
\hline PAS & $.440 * *$ & 1 & $.714 * *$ & $.217^{*}$ & -0.066 & $.563 * *$ \\
\hline PAD & $.533 * *$ & $.714 * *$ & 1 & $.221 *$ & 0.112 & $.438 * *$ \\
\hline ICC & $.426 * *$ & $.217^{*}$ & $.221 *$ & 1 & $.506^{* * *}$ & 0.035 \\
\hline PGC & $.540 * *$ & -0.066 & 0.112 & $.506^{* *}$ & 1 & $-.494 * *$ \\
\hline MME & $.374 * *$ & $.563^{* *}$ & $.438 * *$ & 0.035 & $-.494 * *$ & 1 \\
\hline
\end{tabular}

** La correlación es significativa en el nivel 0,01 (bilateral).

* La correlación es significativa en el nivel 0,05 (bilateral).

IMC: índice de masa corporal. PAS: presión arterial sistólica. PAD: presión arterial diastólica. ICC: índice cintura-cadera. PGC: porcentaje de grasa corporal. MME: masa de músculo esquelético.

Las correlaciones bilaterales de Pearson se expresan en la tabla 8. Se observó una correlación moderada (0.5) entre IMC y PGC, PAS y MME.

\section{DISCUSIÓN}

Se observó mayor participación del género femenino, en un rango de edad comprendido entre los 18 y 26 años. El parámetro con mayor dispersión en los datos correspondió a PAS, rango que se mantuvo dentro de los parámetros que comprenden a hipertensión G1, las cifras más altas se observaron en el género masculino. Respecto a la PAS, la media encontrada en este estudio se encuentra en el límite recomendado $(110 \mathrm{mmHg})$, dato importante, ya que cifras de $10 \mathrm{mmHg}$ mayores implican un $50 \%$ más de riesgo para padecer enfermedad cardiovascular. Estos hallazgos han sido reportados en otros estudios, donde las cifras tensionales altas predominan en el género masculino (Campos et al, 2016). Incluso, se considera a la PAS entre 110-115 mmHg como un factor de riesgo de pérdida de la salud global, conforme a lo señalado por Forouzanfar (2017), quien reportó la pérdida de años de vida ajustados por discapacidad (AVAD) asociados a estos valores. La PAD media encontrada en este estudio fue menor a las recomendaciones emitidas por el estudio Framingham, donde una PAD $<70 \mathrm{~mm} \mathrm{Hg}$ con PAS $\geq 120 \mathrm{~mm} \mathrm{Hg}$ se asoció con un riesgo de ECV equivalente a aproximadamente $20 \mathrm{~mm} \mathrm{Hg}$ de aumento adicional en la PAS (Flanklin y Wong, 2013).

La media en el parámetro de ICC para esta población se encontró por encima del parámetro normal recomendado, por lo cual se infiere que la adiposidad abdominal es un factor predominante en estos individuos. La media de ACT, PGC, MGC, y proteínas también presentan medias fuera de rangos normales. El indicador de minerales por kilogramo es el único parámetro con una media en rango de normalidad. Conforme a lo esperado, el género femenino presento MGC, dato normal en su composición (dimorfismo sexual), siendo el único parámetro superior al género opuesto, datos que se han confirmado en otros estudios (Cossio et al, 2011). Más del 35\% de los estudiados presentan sobrepeso u obesidad, cifra que duplica lo encontrado en poblaciones similares (Ortega, 2017). Estos resultados son de gran interés puesto que se ha observado a mayores valores de masa muscular y menores de grasa corporal pueden asociarse a un mejor rendimiento cognitivo (Carrillo et al, 2021). 
Respecto a sobrepeso y obesidad, los datos proporcionados por ENSANUT 2012 y 2018 (Shamah et al, 2019) se ha observado una tendencia al alza, donde la obesidad predomina en el género femenino. También se reportó que la obesidad abdominal según parámetros de circunferencia de cintura ha aumentado tanto en ambos géneros durante el mismo periodo, aunque en mayor porcentaje en el masculino (3 y 7\%). Barquera et al. (2020) determinaron que 34\% de los adultos entre 20 y 29 años en México presentan sobrepeso y $25 \%$, obesidad, también observó que el género masculino tiene mayor tendencia al sobrepeso (42.2\% vs 36,6\%), y las femeninas mayor tendencia a la obesidad (39.3\% vs 30.6\%). En el presente estudio se encontró una mayor prevalencia de obesidad en el género masculino. Campos et al (2016) observó que entre el 9.6 a 25\% de aquellos que padecen hipertensión arterial en el grupo de 20-29 años, padecen sobrepeso u obesidad.

Yakoob et al. (2016) identificaron a una dieta subóptima como principal causa de mortalidad cardiometabólica en 4 de 5 países asiáticos, identificando como dieta subóptima a aquellas con bajo consumo de frutas, cereales integrales y verduras, el bajo contenido de grasas omega-3 y el alto consumo de sodio, relacionándose a enfermedad cardiovascular isquémica, EVC isquémico y hemorrágico, otras enfermedades cardiovasculares y diabetes. Este hecho fue observado en el presente estudio, donde las comidas rápidas y bebidas carbonatadas se traducen en un alto consumo de sodio y grasas trans, además de un consumo de frutas y verduras inferior a lo recomendable. Esto ha sido corroborado en literatura nacional (Shamah et al, 2019), que ha reportado que los adolescentes sin programas de ayuda alimentaria, la obesidad aumentó 60\% entre 2012 y 2018 debido a este tipo de alimentación. El análisis de la ENSANUT proporcionado por Barquera et al. (2020), encontró que entre 35 a $40 \%$ de la población mexicana con sobrepeso y obesidad también presenta inseguridad alimentaria en diversos grados.

Diferente a lo reportado por Rodríguez et al. (2019) quienes reportan que los estados del sur suelen consumir carne y lácteos con menor frecuencia, el presente estudio encontró que este grupo de alimentos es el más consumido por los universitarios. Al igual, se esperaba encontrar mayor consumo de frutas y leguminosas en esta población, la ausencia o deficiencia de alimentos que proporcionan fibras no digeribles pueden propiciar disbiosis intestinal, lo cual también ha sido identificado como un factor de riesgo importante para el desarrollo de sobrepeso y obesidad (Flores, et al. 2021). Podría considerarse qué, al tratarse de estudiantes universitarios, sus familias cuentan entre sus posibilidades el tener acceso a alimentos de alto valor económico como las carnes y lácteos, cambiando el comportamiento esperado. Sin embargo, conforme a los resultados de ENSANUT 2018, los estados del centro y sur de México presentan mayor consumo de botanas, bebidas endulzadas, dulces y postres, dato similar a lo encontrado en este análisis. El porcentaje de universitarios que consumen comidas rápidas es en extremo superior a lo encontrado por Torres et al (2016). 
Respecto a los hábitos alimentarios negativos encontrados, autores como Shamah, Mundo y Juan (2014) han encontrado que $70 \%$ de los adultos con obesidad y sobrepeso residen en hogares con inseguridad alimentaria. Los alimentos consumidos dependen en diversidad y acceso conforme a las posibilidades económicas de los individuos, por ello, estos hábitos tienen un trasfondo multifactorial. Pese a esto, la ENSANUT 2018 (Shamah et al, 2021) reportó que aquellos que tienen el nivel de licenciatura, tienen mayor posibilidad de seguridad alimentaria, es por esto por lo que se podría considerar que los resultados obtenidos pueden deberse a factores como falta de tiempo y hábitos alimentarios.

Respecto a toxicomanías, Barquera et al. (2020) encontró que aquellos que padecen sobrepeso y obesidad, refieren consumir alcohol y tabaco; los resultados obtenidos en este estudio para dicha variable fueron mínimos.

\section{CONCLUSIONES}

Se identificó que más de la mitad de los estudiantes presentaron antecedentes heredofamiliares para enfermedades crónico degenerativas. Los parámetros de tensión arterial sistólica se encontraron en el límite superior, lo cual se considera alarmante ante la edad de los sujetos estudiados. La tendencia nacional al sobrepeso en hombres y obesidad en mujeres también pudo observarse en este estudio. Los parámetros de ICC observados nos permiten inferir una frecuencia alta de adiposidad abdominal.

El comportamiento en cuanto hábitos alimentarios en esta población difiere de lo esperado conforme a los resultados nacionales previos, lo cual puede explicarse dado que, al tratarse de individuos cursando una licenciatura, es más probable que provengan de familias con mayor seguridad alimentaria.

El alto consumo de alimentos con elevado contenido de sodio, grasas trans y bajo omega-3 aunado al menor consumo de frutas y verduras también fue mayor a lo esperado, lo cual predispone a los estudiantes al desarrollo de enfermedades cardiovasculares a temprana edad.

Las toxicomanías fueron mínimas en la población estudiada. 


\section{REFERENCIAS}

Barquera, S., Hernández-Barrera, L., Trejo, B., Shamah, T., Campos-Nonato, I., \& Rivera-Dommarco, J. (2020). Obesidad en México, prevalencia y tendencias en adultos. Ensanut 2018-19. Salud Pública De México, 62(6, Nov-Dic), 682-692. https://doi.org/10.21149/11630

Basto-Abreu, A., Barrientos-Gutiérrez, T., Rojas-Martínez, R., Aguilar-Salinas, C. A., López-Olmedo, N., De la Cruz-Góngora, V., Rivera-Dommarco, J., Shamah-Levy, T., Romero-Martínez, M., Barquera, S., López-Ridaura, R., Hernández-Ávila, M., \& Villalpando, S. (2019). Prevalencia de diabetes y descontrol glucémico en México: resultados de la Ensanut 2016. Salud Pública De México, 62(1, enefeb), 50-59. https://doi.org/10.21149/10752

Campos-Nonato, I et al. Hipertensión arterial en adultos mexicanos: prevalencia, diagnóstico y tipo de tratamiento. Ensanut MC 2016. Salud Pública de México [online]. 2018, v. 60, n. 3 [Accedido 31 Enero 2022] , pp. 233-243.

Carrillo Ramírez, C., \& Triana Reina, H. (2021). Relación de la composición corporal y la velocidad de procesamiento cognitivo en estudiantes universitarios: un estudio transversal. NOVA, 19(36).

Centro Nacional de Programas Preventivos y Control de Enfermedades (S.F). Estrategia Estatal Para La Prevención y el Control del Sobrepeso, la Obesidad y la Diabetes (p. 6). Tuxtla Gutiérrez: CENAPRECE.

Cossio-Bolaños Ma, De Arruda M, Moyano Portillo A, Gañán Moreno E, Pino López Lm, Lancho Alonso Jl. Nutrición Clínica y Dietética Hospitalaria 2011;31(3): 15-21

Cruz-Rodríguez, J. González-Vázquez, R. Reyes-Castillo, P. Mayorga-Reyes, L. Nájera-Medina, O. Ramos-Ibáñez, N. Rodríguez-Magallanes, M. Díaz-García, R. Azaola-Espinosa, A. (2019). Ingesta alimentaria y composición corporal asociadas a síndrome metabólico en estudiantes universitarios. Revista mexicana de trastornos alimentarios, $10(1), \quad$ 42-52. https://doi.org/10.22201/fesi.20071523e.2019.1.495

Flores, R., Pérez, B., Fernández, G., Galarza, A., Aguiar, L., \& Mendoza, V. (2021). Prevalencia de factores de riesgo que alteran la microbiota intestinal en niños escolares con sobrepeso y obesidad. South Florida Journal Of Development, 2(3), 4706-4718. https://doi.org/10.46932/sfjdv2n3-068

Forouzanfar, M., Liu, P., Roth, G.., Ng, M., Biryukov, S., Marczak, L., ... Akinyemiju, T. F. (2017). Global BurdenofHypertension and SystolicBloodPressureof at Least 110 to $115 \mathrm{~mm} \mathrm{Hg}, 1990-2015$. JAMA, 317(2), 165. doi:10.1001/jama.2016.19043

Franklin S. Wong N. Hipertensión y enfermedad cardiovascular: contribuciones del estudio del corazón de Framingham. Corazón mundial. 2013;8(1):49-57. DOI: http://doi.org/10.1016/j.gheart.2012.12.004

Gómez-Landeros, Ofelia, \& Galván-Amaya, Geraldine del Carmen, \& Aranda-Rodríguez, Ricardo, \& Herrera-Chacón, Carlos, \& Granados-Cosme, José Arturo (2018). Prevalencia de sobrepeso, obesidad y antecedentes de enfermedad crónica en universitarios mexicanos. Revista Médica del Instituto Mexicano del Seguro Social, 56 (5),462-467.

Instituto Nacional de Estadística y Geografía. (2021). Características de las defunciones registradas en México durante el 2020. Preeliminar (pp. 1-5). México: INEGI.

Jiménez Acevedo, H. Núñez Medina, G. (2016). El sistema de salud de Chiapas ante la transición demográfica y epidemiológica. Cuadernos del CENDES, 33(92),79-103. 
Organización Mundial de la Salud (2021). Enfermedades no transmisibles. Who.int. Consultado en 13 enero de 2022, fromhttps://www.who.int/es/news-room/fact-sheets/detail/noncommunicable-diseases.

Ortega GJA, Fernández GOAA, Bilbao RT, et al (2017). Estado de la composición corporal del estudiante de la Facultad de Medicina de una universidad pública de México. RCAN. 2017;27(1):14-28.

Rodríguez-Ramírez, S., Gaona-Pineda, E. B., Martínez-Tapia, B., Arango-Angarita, A., Kim-Herrera, E. Y., Valdez-Sánchez, A., Medina-Zacarías, M. C., Shamah-Levy, T., \& Ramírez-Silva, . I. (2020). Consumo de grupos de alimentos y su asociación con características sociodemográficas en población mexicana. Ensanut 2018-19. Salud Pública De México,62(6, Nov-Dic), 693-703. https://doi.org/10.21149/11529

Shamah-Levy, T. Mundo-Rosas, V. Rivera J. (2014). La magnitud de la inseguridad alimentaria en México: su relación con el estado de nutrición y con factores socioeconómicos. Salud Pública de México, 56(Supl. 1), s79-85. Recuperado en 31 de enero de 2022, de http://www.scielo.org.mx/scielo.php?script=sci_arttext\&pid=S003636342014000700012\&lng=es\&tlng=es.

Shamah-Levy, T., Méndez-Gómez Humarán, I. ., Mundo-Rosas, V. ., Rodríguez-Ramírez, S., \& GaonaPineda, E. B. (2021). Factores asociados con el cambio en la inseguridad alimentaria en México: Ensanut 2012 y 2018-19. Salud Pública De México, 63(3 May-Jun), 350-358. https://doi.org/10.21149/12145

Shamah-Levy, Teresa et al. Sobrepeso y obesidad en población mexicana en condición de vulnerabilidad (2019). Resultados de la Ensanut 100k. Salud Pública de México [online]. v. 61, n. 6 [Acceso 31 Enero 2022] , pp. 852-865.

Torres-Mallma, C. Trujillo-Valencia, C. Urquiza-Díaz, Ana Lucía, Salazar-Rojas, Ronald, \& TaypeRondán, Alvaro. (2016). Hábitos alimentarios en estudiantes de medicina de primer y sexto año de una universidad privada de Lima, Perú. Revista chilena de nutrición, 43(2), 146154. https://dx.doi.org/10.4067/S0717-75182016000200006

Yakoob, M. Y., Micha, R., Khatibzadeh, S., Singh, G. M., Shi, P., ... Ahsan, H. (2016). Impact of Dietary and Metabolic Risk Factors on Cardiovascular and Diabetes Mortality in South Asia: Analysis From the 2010 Global Burden of Disease Study. American Journal of Public Health, 106(12), 2113-2125. doi:10.2105/ajph.2016.303368 